\title{
La médicalisation de la procréation en Transjordanie. Trajectoires féminines à l'époque du Mandat
}

Irene Maffi

\section{Résumé}

L'article porte sur la médicalisation de la procréation promue par le gouvernement colonial en Transjordanie et la transformation des pratiques de la naissance au sein de la société locale. Il analyse en particulier l'impact des politiques sanitaires sur l'existence des femmes colonisées, en se penchant tant sur la manière dont elles ont contribué à transformer les pratiques procréatives que sur les nouvelles trajectoires de vie des femmes qui choisirent de s'engager dans les professions de santé. Les soignantes qui embrassèrent le système sanitaire imposé par les colonisateurs devinrent en même temps objets et agents de changements sociaux qui, à long terme, contribuèrent à transformer la manière de penser la naissance, la parentalité et la famille.

\begin{abstract}
The article focusses on the medicalisation of procreation promoted by the colonial governement in Transjordan and on the consequent transformation of birth practices in the local society. It specifically investigates the impact of public health policies on colonised women's lives considering the ways the former changed local procreation practices and the new life trajectories they elicited for the women who chose to train as birth attendants. Transjordanian midwives and nurses trained in the newly imposed biomedical
\end{abstract}

Irene Maffi, Laboratoire d'anthropologie culturelle et sociale, Institut des sciences sociales, Faculté des sciences sociales et politiques, Université de Lausanne, CH-1015 Lausanne, irene.maffi@unil.ch. 
system became at the same time objects and agents of social change contributing in the long term to the transformation of birth, parenting and family representations.

Keywords: birth attendants, colonisation, medicalisation, reproduction, Transjordan

\section{Les services de santé maternelle et infantile à l'époque du Mandat britannique (1921-1946)}

Le premier petit hôpital (21 lits) destiné aux femmes et aux enfants fut créé dans l'Emirat de Trasjordanie en 1927 par la Doctoresse Charlotte Purnell, membre de la Church Missionary Society (CMS), une association caritative de l'Eglise anglicane active dans plusieurs colonies britanniques. Appelée par la population locale «la dame boîteuse» à cause d'une blessure qu'elle s'était procurée en tombant de cheval lors d'une visite chez une patiente qui résidait dans une région éloignée, elle utilisa ses propres fonds pour financer la création de la première maternité du pays. ${ }^{1}$

En Transjordanie, la CMS ouvrit deux cliniques pour la protection maternelle et infantile (PMI) qui, avec l'hôpital de la Dresse Purnell, furent, à quelques exceptions près, ${ }^{2}$ les seuls centres actifs jusqu'à la fin des années $1940 .{ }^{3}$

A l'époque du Mandat britannique sur la Palestine et la Transjordanie, ${ }^{4}$ les quelques médecins (femmes), infirmières et sages-femmes européennes présentes dans la région jouèrent un rôle important dans la médicalisation de la procréation puisque, étant considérées comme «naturellement les plus adaptées pour prendre soin de leur propre sexe», ${ }^{5}$ le domaine de la santé maternelle et infantile leur était réservé. La création d'un espace féminin séparé au sein des institutions sanitaires doit être mise en relation avec l'idéologie répandue en Europe selon laquelle la mise au monde, l'élevage, l'éducation des enfants et la gestion de l'hygiène domestique étaient des affaires

1 Sultan 1998.

2 Il faut mentionner l'Hôpital italien fondé à Amman en 1922, qui toutefois n'avait pas un service d'obstrétrique et gynécologie, et un hôpital public (20 lits) fondé à Amman en 1939 comprenant un petit département d'obstétrique. A l'aube de la Seconde Guerre mondiale, les hôpitaux de l'Emirat étaient au nombre de 17, dont 5 gérés pas des sociétés missionnaires (l'Hôpital italien à Amman, l'Hôpital italien à Karak, l'Hôpital de la CMS à Salt, l'Hôpital de la CMS à Ajloun) un par un médecin de libre profession (l'Hôpital Malhas à Amman) pour un total de 124 lits; et 12 hôpitaux gouvernementaux pour un total de 169 lits (Annual Report of the Department of Health of Transjordan 1939).

3 Pour plus de détails, voir Maffi 2012.

4 Palestine et Transjordanie faisaient partie d'une même entité politique soumise à l'administration britannique.

5 Arnold 1993, 265. 
de femmes. En outre, la très grande majorité des médecins européens étaient des hommes et les normes locales de la bienséance empéchaient le contact physique entre eux et les femmes transjordaniennes. ${ }^{6}$

Les deux centres de PMI créés par la CMS recevaient de maigres sommes du département de la Santé de l'Emirat de Transjordanie (DSET), leur permettant à peine de payer le salaire d'une infrimière à Amman et de couvrir le coûts des médicaments à Salt, bien que dans les années 1920 cette dernière fût la ville la plus importante de l'Emirat. ${ }^{7}$ A cause du manque de personnel, le centre de PMI de Salt ferma ses portes à plusieurs reprises au cours des années 1920 et 1930 en fonction de la présence de médecins ou infirmières bénévoles. Par exemple, en 1929, il fut fermé pendant neuf mois lorsque l'infirmière de la CMS en charge de celui-ci partit pour la Grande-Bretagne. ${ }^{8}$

Pour comprendre la situation de la Tramsjordanie pendant l'époque mandataire, il est intéressant de citer les mémoires du Dr Jamil Al-Tutunji, le premier médecin de l'Emir Abdallah, dans lesquelles il raconte ses visites dans le Sud du pays pendant les années 1918-1921. Il décrit les pénibles conditions de voyage -asbence de voies de communication, de moyens de transports et d'auberges - e les difficiles relations avec les habitants de cette région. Il souligne qu'il était presque impossible d'examiner, voire de rencontrer les femmes, et que les hommes étaient très méfiants à son égard, refusant même de répondre à ses questions. ${ }^{9}$ La méfiance de la population locale vis-à-vis des nouvelles pratiques médicales peut être expliquée en partie par le fait qu'au début des années 1920, dans l'Emirat il n'y avait que 5 médecins formés en biomédecine et qu'ils vivaient dans les rares aires urbaines. ${ }^{10}$ Le nombre très restreint de professionnels de la santé présents en Jordanie est attesté par les les rapports annuels de l'adminsitration britannique aux Nations Unies (RAAPT) qui constituent la source principale permettant de retracer l'évolution des institutions sanitaires en Transjordanie. Par exemple, le RAAPT de 1934 indique que de nouvelles licences avaient été octroyées à 6 médecins, 2 dentistes, 2 sages-femmes et un pharmacien; selon le RAAPT de 1937, les médecins exerçant dans l'Emirat étaient 19 (y compris les officiers médicaux de l'armée) et les sages-femmes agrées 11 alors que la population dépassait les 300000 habitants.

6 Rogan 1999; voir aussi le témoignage du Dr Jamil Al-Tutunji cité plus bas.

7 Au début des années 1920, la population totale de l'Emirat était de 225380 habitants, dont 20000 résidaient dans le district de Salt. 46 pour cent de la population était classé comme nomade (Wilson 1987: 55-56). En 1950, les habitants de la Jordanie étaient 476000 (ibid: 198).

8 Rapport adminsitratif annuel sur la Palestine et la Transjordanie (RAAPT) 1932.

9 Al-Tutunji 1957.

10 Mahafza 1990. 
La Transjordanie du début du $20^{\mathrm{e}}$ siècle était une région désertique située aux marges des riches provinces ottomanes de Palestine et de Syrie où il y avait de petits centres urbains situés surtout dans le Nord du pays. Malgré les relations commerciales que ces centres entretenaient avec les villes palestiniennes et syriennes, la plupart de la population vivait de l'agriculture et de l'élevage d'animaux. ${ }^{11}$

L'exiguïté des services sanitaires destinés aux mères et aux enfants pendant l'époque du Mandat est à mettre en relation avec, d'une part, les ressources très restreintes dont disposaient l'Emirat et le DSET en particulier, ${ }^{12}$ d'autre part, la résistance de l'Etat britannique à considérer la santé des femmes comme relevant de sa responsabilité. ${ }^{13}$ Ces deux aspects expliquent la présence de médecins, infirmières et sages-femmes travaillant dans des institutions caritatives engagées dans le domaine de la santé maternelle et infantile et l'absence de structures publiques. En Transjordanie, comme en Palestine et dans d'autres colonies britanniques, les centres de soins maternels et infantiles furent créés surtout par des institutions religieuses et gérées par des missionnaires. Les objectifs de ces dispensaires étaient non seulement d'offrir des soins médicaux aux femmes et aux enfants, mais également de «civiliser» la population locale, lui transmettant des connaissances «modernes», et de la convertir au christianisme, «moralisant» ses conduites sexuelles et ses coutumes familiales. L'administration coloniale profita de cette situation pour déléguer le financement des équipements et du personnel des structures sanitaires aux sociétés missionnaires, promouvant ainsi une «entreprise conjointe $»^{14}$ dans laquelle elle se limitait à offrir un soutien logistique et financier minimal.

Une des préoccupations des Britanniques en Transjordanie consistait à diminuer la mortalité infantile ${ }^{15}$ plusieurs fois décriée dans les rapports annuels fournit aux Nations Unies, car mettant en danger le développement socioéconomique du pays. ${ }^{16}$ Malgré des considérations sur les conditions de

11 Rogan et Tell 1994.

12 Amadouni 1994.

13 Arnold, 1993, 268.

14 Koven et Michels 1993.

15 Les taux de mortalité infantile étaient difficiles à calculer car le nombre exact de la population n'était pas connu et les familles enregistraient rarement leurs enfants à l'état civil une institution coloniale créée en 1918. Le RAAPT de 1925 indique qu'en Palestine la mortalité était de 180 enfants pour 1000, bien que, comme indiqué dans le RAAPT de 1932, les taux oscillent très fortement d'une année à l'autre selon les enregistrements effectués à l'état civil. Ainsi, en 1930 en Palestine la mortalité était de 154 pour mille tandis qu'en 1931 elle était de 170 pour mille. Dans la section des rapports dédiée à la Transjordanie, la mortalité infantile n'était pas indiquée de manière systématique: dans le RAAPT de 1934, elle était estimée à 242 pour mille et dans celui de 1938 à 181 pour mille.

16 Amadouni 1997. 
pauvreté, de malnutrition et de manque d'hygiène dans lesquelles vivait la population locale, dans les rapports coloniaux la responsabilité de la mortalité infantile élevée était principalement attribuée aux femmes. Les fonctionnaires mettaient l'accent sur le fait que les mères ignoraient les règles fondamentales pour l'élevage des enfants et, qui plus est, faisaient appel aux matrones ou aux accoucheuses locales pour mettre au monde leurs enfants. Les pratiques des dayat ${ }^{17}$ (accoucheuses) et des matrones étaient définies comme «barbares», «sales» et «dangereuses» et fortement stigmatisées. Considérées avec les mères comme les principales responsables de la mortalité infantile, les dayat devinrent la cible des réformes dans le domaine de la santé maternelle et infantile. ${ }^{18}$ Ces réformes visaient à réglementer, sinon à éradiquer, les pratiques locales de la naissance d'une part et à éduquer les mères pour qu'elles s'occupent de manière appropriée de leurs enfants de l'autre.

Les documents coloniaux reflètent des opinions largement partagées au sein de l'administration britannique aussi bien que des milieux missionnaires engagés auprès de la population transjordanienne. Le témoignage de Madame Lampitt qui travailla dans le centre de PMI de Salt, l'illustre clairement:

The work at this Centre was commenced in January 1928, by Miss E. Lester, in a room on the Jedda Jill, at first only 2 or 3 mothers could be persuaded to attend, and were afraid of evil happening to their children if they were weighed, but by the end of May there were 8 or more attendances each afternoon twice a week, and some of the homes were visited. Simple teaching was given and before Miss Lester left for England the mothers were questioned as to their knowledge and a prize given for the best answers, also for the cleanest baby. ${ }^{19}$

Madame Lampitt, comme les autres membres du personnel travaillant dans les institutions missionnaires, traitait les mères comme des enfants à éduquer pour qu'elles assimilent les connaissances biomédicales et les normes d'hygiène en vigueur en Europe. Une pratique courante en Transjordanie et Palestine était celle de donner des prix sous forme d'encouragements matériels aux femmes qui appliquaient les préceptes qui leur avaient été enseignés. ${ }^{20}$ Les services offerts dans les centres de PMI étaient très basiques: des consultations générales, la pesée des enfants et l'éducation des mères, en particulier concernant l'hygiène et l'alimentation, parfois la distribution de savon ou d'aliments. ${ }^{21}$

17 Dayat est le pluriel de daya.

18 Pour des discours similaires dans d'autres colonies britanniques voir par exemple: Arnold 1993; Boddy 2007; Hugon 2005; 2011; Jolly et Ram 1998; Van Hollen 2003.

19 Cité dans Young 1997, 101.

20 A ce propos voir par exemple Hunt 1997; Summers 1991; Van Tol 2007.

21 Annual Report of the Department of Health of Transjordan 1929. 
Le but de cet article n'étant pas de brosser un tableau exhaustif de l'évolution des politiques de santé maternelle et infantile à l'époque coloniale, je vais me pencher sur le rôle joué par quelques figures de femmes transjordaniennes et palestinienne ${ }^{22}$ dans la construction d'un nouveau «système de naissance ${ }^{23}$ et sur son impact sur les pratiques reproductives au sein de la société locale. Afin de situer leurs trajectoires de vie dans le contexte historique de l'époque, je vais décrire quelques-unes des transformations que l'administration britannique causa dans l'existence des femmes colonisées, et plus largement de la société transjordanienne. Outre les changements des pratiques domestiques et familiales, la création de services de santé destinés aux mères et aux enfants contribua à déterminer l'accès des femmes locales aux professions de la santé modifiant leur statut et leur rôle social. Les mères, les soignantes et les dayat qui s'adaptèrent au système de naissance imposé par les colonisateurs devinrent en même temps des objets et des agents de changements qui à long terme contribuèrent à transformer la manière de penser la naissance, la parentalité et la famille. Afin de montrer l'impact des institutions sanitaires introduites par les Britanniques, je vais d'abord esquisser les formes d'adaptation que les dayat durent mettre en place pour garder, au moins en partie, leur rôle dans la société locale. Je décrirai ensuite les itinéraires professionnels des deux premières sage-femmes qui se formèrent dans les institutions médicales coloniales. En conclusion, j'analyserai les éléments nouveaux que leur parcours personnel et professionnel révèle et mettrai en lumière les transformations dont ils sont porteurs.

\section{Les dayat: actrices ou objets de changement?}

A cheval entre le $19^{\mathrm{e}}$ et le $20^{\mathrm{e}}$ siècles, le mot dayat désignait les femmes possédant des connaissances et des techniques dans le domaine de la procréation et de la santé féminine: elles surveillaient les accouchements, s'occupaient des bébés et des mamans et étaient responsables de tous les moments rituels liés à la vie reproductive des femmes et à la naissance des enfants. Pour devenir daya, une femme ne devait pas suivre une éducation formelle, mais acquérait ses compétences en suivant une accoucheuse experte qui lui apprenait l'art par compagnonnage. Souvent, l'art des accoucheuses était transmis au sein de la famille: la mère ou une parente proche transmettait ses connaissances à l'apprentie daya. Les dayat étaient aussi des spécialistes des

22 Emirat de Transjordanie est le nom de l'Etat jordanien à l'époque du Mandat britannique. Après l'Indépendance (1946), il pris le nom de Royaume hachémite de Jordanie.

23 Traduction de l'expression «birth system» créée par Brigitte Jordan (1993). 
remèdes à base de plantes et pouvaient soigner diverses maladies parmi lesquelles la stérilité, la carence de lait pendant l'allaitement, les suites des fausses couches, les infections, etc. Il est difficile de brosser un portrait précis des dayat, dans la mesure où leur statut social, leurs connaissances, leurs techniques et leur formation variaient d'un contexte à l'autre. Dans les aires urbaines et dans certains villages, elles étaient des figures spécialisées jouissant d'un statut social relativement élevé, tandis que chez la population nomade et semi-nomade, le rôle d'accoucheuse était joué par toute femme âgée ayant mis au monde des enfants. Comme mentionné plus haut, après la création de l'Emirat de Transjordanie, les pratiques de la naissance en vigueur durant les dernières décennies de l'empire ottoman furent combattues par les fonctionnaires coloniaux. Un processus de réglementation des pratiques reproductives fut mis en place amenant à la mise sur pied d'un office de l'état civil pour enregistrer les naissances, à l'enregistrement systématique des dayat et à la création de programmes de «ré-éducation» destinés aux accoucheuses. Dès la moitié des années 1920, les autorités britanniques décidèrent également de règlementer les professions de la santé imposant l'inscription des membres des divers corps - médecins, dentistes, sagesfemmes, infirmières, pharmaciens - dans les registres officiels. ${ }^{24}$ Malgré ces mesures, le système de naissance local ne pouvait pas être démantelé en quelques jours: sa suppression dut passer par différentes phases qui amenèrent à la limitation de plus en plus importante des prérogatives des dayat et à la dévaluation progressive de leur savoir et de leurs pratiques.

En 1922, un centre de formation pour les sages-femmes agréées fut créé au sein du Jerusalem Governement Hospital où venait d'être inauguré un département d'obstétrique et gynécologie, grâce à des dons recoltés en l'honneur de sa Majesté. ${ }^{25}$ La création du centre avait été encouragée suite à la dénonciation du RAAPT de 1921, selon lequel:

Midwifery in Palestine is almost unsatisfactory, The 51 qualified women are confined to a few of the larger towns and there are no less than 884 untrained and, in most cases, grossly ignorant, practising midwives registered at the District Health Offices. There are few problems more urgent than the establishment of centres for the teaching of midwifery and infant management (ibid: 271).

En 1929, une ordonnance fut promulguée afin de réglementer la formation et la pratique des sages-femmes en Palestine et Transjordanie. Ces dernières devaient être formées dans des centres médicaux reconnus en accord avec les standards établis par le British Central Midwifery Board. Si le projet colonial 
était d'éradiquer les dayat, le manque de sages-femmes agréées pour les remplacer obligea les autorités britanniques à reporter la suppression des accoucheuses locales. Dans la période de transition, des cours de «rééducation» et de formation furent rendus obligatoires pour celles qui voulaient continuer à exercer légalement afin de lutter contre leur «ignorance» et «les soufrances inutiles» dérivant de leurs pratiques. ${ }^{26}$ Le processus amenant au remplacement des dayat par les sages-femmes agréées fut plus rapide en Palestine où en 1939, les 1239 accoucheuses enregistrées étaient autorisées à exercer seulement dans les aires où les sages-femmes agréées n'étaient pas présentes c'est-à-dire principalement dans les régions rurales. ${ }^{27}$

En Jordanie, le processus d'élimination définitive des dayat ne put être accompli que durant la période postcoloniale par l'Etat indépendant qui poursuivit les politiques entreprises par l'administration britannique. ${ }^{28}$ Quand à la moitié des années 1990, l'anthropologue américaine Elise Young effectua sa recherche de terrain ${ }^{29}$ dans le camp de réfugiés palestiniens de Baq'a, situé au nord d'Amman, les dayat pratiquaient encore et étaient enregistrées auprès de l'UNRWA. ${ }^{30}$ Young récolta les témoignages de plusieurs dayat palestiniennes qui arrivèrent en Jordanie comme réfugiées après 1948 et qui lui racontèrent les transformations de leur métier entre les années 1940-1980. Le travail de Young est unique dans la mesure où il constitue la seule étude donnant la voix aux dayat, dont nous ne possédons que l'image négative transmise par les documents coloniaux et les témoignages des missionnaires ${ }^{31}$ Les interlocutrices de Young décrivent leur perception des changements provoqués par les règles imposées par l'administration coloniale d'abord et adoptées par les gouvernements postcoloniaux après, permettant d'accéder à la perspective de ce groupe de femmes. Le changement et parfois la destruction de leur univers incarnent les transformations plus profondes qui ont touché les sociétés palestinienne et transjordanienne durant les années de formation des Etats nations modernes. Les dayat de l'ancienne génération ${ }^{32}$ décrivent l'instauration de nouveaux rapports de pouvoir entre

26 RAAPT 1932, 137-8.

27 RAAPT 1939.

28 En 1938, les sage-femmes agréées exerçant en Transjordanies étaient 9 (RAAPT 1938).

29 Young 1997.

30 La United Nations Relief and Works Agency est l'agence onusienne en charge des réfugiés palestiniens.

31 Des descriptions ethnographiques des pratiques de la naissance en Palestine et Transjordanie au début du $20^{\mathrm{e}}$ siècle se trouvent aussi dans les travaux de Granqvist 1947 et Jaussen $1908 ; 1927$.

32 Les dayat de l'ancienne génération avaient commencé à pratiquer pendant les dernières années du Mandat britannique (début des années 1940). Elles étaient donc les dernières représentantes d'une figure en train de disapraître. En Jordanie, les cours de formation pour les accoucheuses continuèrent à être donnés pendant plusieurs décennies après 
elles et les professionnels de la santé avec une formation biomédicale, le changement de statut des accoucheuses, les effets économiques de la médicalisation de la procréation, l'imposition d'un fort contrôle administratif sur la vie reproductive des colonisés, etc. Leurs discours révèlent des points de vue qui ne peuvent être retrouvés dans les documents officiels ni dans les témoignages des sages-femmes agréées et des médecins qui les côtoyèrent, dont le point de vue est fortement influencé par la logique moderniste du paradigme biomédical. Ils montrent également l'impact négatif des campagnes de diffamation lancées à leur encontre par les administrateurs coloniaux et l'effet durable de ces discours qui continuèrent à modeler les politiques de santé de l'Etat postcolonial.

Un des sujets qui émerge des discours des dayat du camp de Baq'a concerne leurs relations avec le savoir et les pratiques biomédicaux qu'elles appréhendaient de manière ambivalente. Certaines accoucheuses - qui avaient suivi les cours de formation dans les hôpitaux - se plaignaient que la certification imposée par l'Etat avait dévalué leur rôle auprès des femmes, tandis que d'autres étaient satisfaites de ce qu'elles avaient appris pendant les cours et collaboraient volontiers avec les médecins. ${ }^{33}$

Toutefois, les dayat et les mères, bien qu'appréciant les avantages inhérents au modèle biomédical, n'étaient pas prêtes à embrasser le nouveau système dans son intégralité, préférant sélectionner les aspects qu'elles percevaient comme positifs. ${ }^{34}$ Par exemple, une daya insistait sur le fait que:

Women come when they don't want to get stitched and they don't want to have the opening ... the midwife does not give her medicine to hasten contractions, she gives them all the time they need, and if that doesn't work and the position of the baby is not correct she takes them to the hospital. The doctors pressure women, but the midwife does not leave her. ${ }^{35}$

Les dayat du camp de Baq'a se plaignaient également de l'absence d'un nombre suffisant de centres médicaux et du coût élevé des traitements, éléments qui empêchaient les femmes d'y avoir recours les poussant à s'adresser à des thérapeutes non allopatiques. De fait, à partir de l'époque coloniale, le pluralisme médical devint courant comme le montrent le récit autobiographique d'Abdul-Rahman Munif (1998) pour la Transjordanie et l'étude de Naomi Shepherd (1999) sur la Palestine. Pendant la seconde moitié du

l'indépendance. J'ai pu rencontrer des sages-femmes agréées qui avaient donné ces cours dans les hôpitaux gouvernementaux dans les années 1960 et 1970 (Maffi 2012).

33 Les attitudes ambivalentes des accoucheuses jordaniennes se retrouvent par exemples chez les accoucheuses maya étudiées par Jordan 1993; Quattrocchi 2011; Sargent et Bascope 1996.

34 Sur la nature sélective des femmes et le pluralisme médical dans un autre pays arabe voir par exemple Inhorn 1996.

35 Young 1997, 122. 
$20^{\mathrm{e}}$ siècle, le recours à différents traitements inscrits dans des modèles thérapeutiques et culturels d'origine diverse était encore la norme, comme le montre l'étude de Muhammad Tbaishat menée dans une ville de la Jordanie septentrionale. ${ }^{36}$ Ici, au début des années 1990, plusieurs catégories de thérapeutes pratiquant des médecines locales coexistaient avec les institutions biomédicales modernes et les professionnels de la santé formés dans les écoles gouvernementales. De manière similaire à ce qui arriva aux dayat et à leurs «clientes», si la biomédecine était valorisée par les habitants de la ville, ceux-ci appréciaient encore les méthodes «traditionnelles» et continuaient à y avoir recours, malgré la disponibilité de thérapies biomédicales. Tant à l'époque coloniale qu'après l'indépendance, le recours au pluralisme médical fut fortement stigmatisé par les autorités étatiques, parce que la médecine arabe et les autres pratiques thérapeutiques locales étaient considérées comme incompatibles avec la biomédecine.

Les relations de pouvoir entre les dayat et le personnel médical et paramédical formé dans les institutions étatiques constituaient un autre sujet abordé par les accoucheuses interviewées par Young. Elles se sentaient en concurrence avec les médecins dont elles remettaient ouvertement en question les pratiques. Une daya racontait l'histoire suivante:

Once I referred a woman in her seventh month to a doctor. She was having dizzy spells. The doctor told her who do you see, and she told him that she is seeing a midwife. The doctor told her, what are you crazy to have a midwife take care of you. She told him that the midwife is the one who sent me to you, and if you don't accept that I will not come to you. ${ }^{37}$

Dans leurs récits, certaines dayat soulignaient comment, malgré des situations très difficiles, elles avaient réussi à sauver une femme dont la vie avait été sérieusement mise en danger par l'intervention (inappropriée) d'un médecin. Tous ces discours exprimaient un conflit de pouvoir entre elles et les professionnels de la santé, dans la mesure où ces derniers étaient identifiés comme les représentants d'un système de santé dépréciant leur savoir et leurs pratiques et mettant en danger l'univers social dans lequel elles étaient inscrites.

Si les dayat pratiquaient encore dans les camps de réfugiés en Jordanie au début des années 1990, dix ans plus tard, elles représentaient des survivances d'un passé révolu, comme l'affirmait Layla, une sage-femme agréée jordanienne qui, entre 1979 et 1999, avait été responsable des centres de PMI dans les camps situés autour d'Amman..$^{38}$ Avant les années 1990, les dayat étaient soumises à des contrôles réguliers et étaient autorisées à pratiquer exclusive-

36 Tbaishat 1991.

37 Young 1997, 122.

38 Maffi 2012. 
ment sous le contrôle du personnel médical de l'UNRWA. ${ }^{39}$ Leurs instruments étaient vérifiés de manière régulière et elles devaient respecter des procédures administratives qui leur imposaient par exemple de remplir des certificats de naissance officiels pour les enfants qu'elles aidaient à naître..$^{40}$ Elles recevaient un salaire mensuel de l'UNRWA mais avaient le droit d'assister seulement aux accouchements des femmes classées par les médecins comme «à bas risque». Selon Layla, les dayat opposaient de fortes résistances à leur incorporation dans le système biomédical institutionnalisé: il y avait encore des accoucheuses «non agréées» qui pratiquaient dans les camps et parfois les dayat reconnues assistaient aux accouchements de femmes «à haut risque».

\section{Les pionnières: Anisa Shuqayr et Nabiha al-Wirr}

La première génération de sages-femmes qui reçut une formation biomédicale au sein d'institutions étatiques - que l'historien jordanien Fathi Sultan dénomme «les pionnières» ${ }^{41}$ - est née dans les années 1910-1920. Juste quelques années avant ce groupe de sages-femmes, est née Anisa Shuqayr, figure fondatrice de l'histoire de l'obstétrique jordanienne, qui a marqué les mémoires de beaucoup de familles d'Amman. Appelée d'habitude par le titre honorifique de hajja Anisa, elle était née à Naplouse en 1905 et s'était mariée à l'âge de onze ans avec un homme de vingt ans plus âgé qu'elle. En 1925, elle suivit une formation de six mois à l'Hôpital Maskubiyya de Jérusalem pour devenir sage-femme agréée. En 1926, elle commença à exercer la profession de sage-femme à Hebron où elle était «très prisée et recherchée» ${ }^{42}$ et, en 1927, devint la première sage-femme municipale d'Amman, fonction qu'elle garda jusqu'en 1948, lorsqu'elle choisit de pratiquer comme indépendante. En 1953, elle s'établit en Arabie saoudite où elle pratiqua la profession de sage-femme pendant cinq ans. Revenue en Jordanie, elle eut encore le temps d'assister à son dernier accouchement en 1978.

Pendant la période mandataire, elle fut présente à la naissance de plusieurs futurs ministres, hommes politiques et personnalités jordaniens ainsi que de citoyens ordinaires. Sultan brosse un tableau très élogieux de hajja Anisa, qu'il présente comme une sage-femme luttant contre «l'ignorance» et

39 La United Nations Relief and Works Agency est l'agence onusienne en charge des réfugiés palestiniens.

40 Ces procédures reproduisent celles mises en place é l'époque coloniale.

41 Sultan 1998.

42 Young 1997, 115. 
le «préjudice» diffus à cette époque au sein de la société transjordanienne. Il raconte divers épisodes qui mettent en lumière l'engagement de la première sage-femme «moderne» de l'histoire jordanienne, celle qui ouvrit la voie aux nouvelles générations «luttant contre l'ignorance de son temps».43

Une autre figure presque mythique de l'histoire de l'obstétrique en Jordanie est Nabiha Salama al-Wirr. Née à Salt en 1915, elle fréquenta d'abord l'Ecole française des Sœurs du Rosaire, puis l'Ecole de la CMS. Une de ses enseignantes l'encouragea à poursuivre sa formation à l'hôpital gouvernemental de Jérusalem, où il y avait une carence chronique de sages-femmes et infirmières. Malgré la résistance initiale de sa famille, en 1929 son père accompagna Nabiha à Jérusalem où elle étudia pendant trois ans pour devenir infirmière. ${ }^{44}$ Dans sa volée, il n'y avait que quatre étudiantes parmi lesquelles une seule arabe, Asma Haddad. En 1932, Nabiha devint infirmière cheffe et en 1935 commença à travailler à l'Hôpital de Haïfa. A ce moment, Mrs Cooper, la responsable du personnel paramédical de l'hôpital, lui demanda si elle était prête à suivre une formation de sage-femme afin de pouvoir assurer ce rôle au sein de l'établissement. Nabiha accepta et en 1936 retourna à l'hôpital al-Maskubiyya de Jérusalem pour prendre le diplôme de sage-femme. Au bout des six mois de stage, elle revint à Haïfa et devint sage-femme cheffe de la maternité de la ville. Elle retourna en Transjordanie en 1945, lorsqu'elle fut nommée responsable du centre gouvernemental de PMI de Zarqa, une ville située au nord de la capitale, pour fournir une assistance médicale aux familles des soldats des Forces transjordaniennes des frontières. En 1948, elle fut nommée par Misbah, la femme du roi Abdallah I, en tant que responsable du département de l'hôpital public de Zarqa en charge des blessés de la Première Guerre contre l'armée sioniste. Une année plus tard, le Dr. Yousef Shuayhat, haut responsable du département de la Santé, lui demanda de superviser la fondation de la première maternité publique d'Amman. Pendant trois ans, Nabiha et sa sœur Maryam - qui était aussi sage-femme - se consacrèrent à la mise en place de l'hôpital pour qu'il puisse accueillir les femmes et leurs bébés. En 1950, elle fut la première femme à faire partie du comité jordanien qui participa au Congrès international de la Croix rouge et du Croissant rouge à Damas. La même année, elle reçut une bourse pour aller à Londres et devenir sage-femme formatrice et à partir de 1951 commença à donner des cours à la première génération de sages-femmes qui se forma dans la nouvelle maternité

43 Sultan 1998, 498-9.

44 La première école de sages-femmes fut instituée en Jordanie après l'indépendance (Sultan 1998). Toutes les sages-femmes qui travaillèrent en Jordanie pendant l'époque mandataire s'étaient formées dans les hôpitaux palestiniens ou ailleurs. 
d'Amman. Les cours duraient deux ans et, en 1953, la première volée de sages-femmes entièrement formées en Jordanie obtint son diplôme. Néanmoins, la première véritable Ecole de sages-femmes ne fut créée qu'en 1956 avec le soutien de l'UNICEF et de la coopération américaine (Point Four Aid Programm). De manière similaire à Anisa Shuqayr, à la fin des années 1950, Nabiha al-Wirr quitta la Jordanie pour s'établir dans les pays du Golfe. En 1959, elle s'installa au Koweït où elle devint responsable de la mise sur pied du réseau des centres de PMI du pays et infirmière cheffe de l'Hôpital royal. Elle forma également la première génération de sages-femmes koweïtiennes qui assumèrent la responsabilité de la gestion des centres de PMI locaux, lorsqu'en 1979 Nabiha quitta le pays pour retourner en Jordanie. Pendant sa longue carrière, elle reçut plusieurs prix et médailles parmi lesquels la médaille Florence Nightingale qui lui fut octroyée par la Croix rouge internationale en reconnaissance de ses mérites professionnels.

\section{La carrière de sage-femme et la transformation du statut des femmes}

De ces deux histoires de vie émergent divers éléments emblématiques qui incarnent en quelque sorte une génération et un univers disparus. En premier lieu, pendant les années 1920-1930, devenir sage-femme ou infirmière était une opportunité presque unique pour les femmes transjordaniennes et palestiniennes de faire une carrière professionnelle et atteindre une certaine indépendance économique et sociale. Hajja Anisa et Nabiha al-Wirr firent une partie de leurs études dans une école éloignée du lieu où vivaient leurs familles et eurent la possibilité de prendre des décisions révélant un certain degré d'autonomie. Il est difficile d'établir si ce fut leur parcours éducatif, leur milieu familial ou leur personnalité à déterminer leur trajectoire de vie. Anisa Shuqayr avoua qu'elle avait décidé de devenir sage-femme parce que «elle voulait échapper à sa réalité - dépendance de son mari et pression pour mettre au monde des enfants à un jeune âge». ${ }^{45}$ Il n'est pas étonnant de constater qu'il y avait un nombre élevé de sages-femmes et d'infirmières qui restaient célibataires, dans la mesure où leur activité professionnelle ne leur permettait pas d'accomplir les tâches domestiques généralement confiées aux épouses et mères de famille. Leur travail absorbait la plupart de leur temps et comportait la possibilité d'être déplacées d'un hôpital à un autre, rendant difficile de concilier leur profession avec la vie familiale. Entreprendre la profession de sage-femme ou d'infirmière signifiait embrasser un destin social 
très différent de celui des autres femmes, pour lesquelles le mariage et la maternité constituaient les accomplissements ordinaires de l'existence. Les carrières professionnelles d'Anisa et Nabiha représentent des trajectoires féminines extraordinaires allant à l'encontre des normes socioculturelles de l'époque. Elles permettent de se pencher sur des aspects de la vie ordinaire de la population transjordanienne et palestinienne de la première moitié du $20^{\mathrm{e}}$ siècle, en montrant qu'elle était en train de connaître des transformations profondes affectant son tissu social et familial. Les biographies d'Anisa et Nabiha permettent également de montrer que le système colonial, malgré ses effets destructeurs, donna la possibilité à certaines femmes de modifier leur rôle et leur statut social. ${ }^{46}$ Les effets du nouvel ordre colonial se manifestèrent au sein de différentes couches de la population: des femmes des classes aisées ainsi que des femmes appartenant à des milieux sociaux plus modestes purent accéder à des sphères professionnelles nouvellement créées et modeler leur existence de manière inédite. Bien que la plupart des dayat - qui étaient majoritairement analphabètes - aient pâti de l'introduction du système public de santé imposé par les Britanniques, certaines d'entre elles réussirent à s'adapter aux nouvelles règles. Elles durent néanmoins accepter de se soumettre à la supervision de l'administration coloniale qui leur imposa de prendre des cours de formation réguliers, limita les zones dans lesquelles elles avaient le droit de pratiquer, modifia leur statut, introduisant des contraintes économiques et administratives. Si les dayat vécurent l'effritement de leur rôle social, plusieurs jeunes femmes célibataires, auxquelles la création des écoles missionnaires et de l'Etat mandataire avait ouvert les portes de l'instruction, eurent la possibilité de changer le cours de leur vie, embrassant une carrière qui les éloigna de la sphère domestique et de la protection familiale ordinaires. Il est remarquable que, malgré l'infraction aux normes sociales et leurs maigres moyens, les familles d'Anisa et Nabiha financèrent leurs études, l'équipement et les uniformes hospitaliers. Beaucoup de sages-femmes et infirmières de la première génération appartenaient à des familles modestes pour lesquelles faire étudier leurs filles signifiait un effort économique important. Puisque les femmes des milieux moins privilégiés étaient d'habitude analphabètes et destinées à se marier et devenir mères, l'investissement des familles dans leur formation entraîna des changements majeurs. Les logiques familiales, le statut et le rôle des femmes changeaient et de nouvelles possibilités s'ouvraient à elles, dans la mesure où

46 Mon analyse sur les effets paradoxaux de la colonisation sur les femmes transjordaniennes rejoint ce qu'ont relevé d'autres travaux portant sur le monde arabe. Voir par exemple Abu Lughod 1998. 
elles pouvaient parcourir des trajectoires de vie différentes de celles qui leur étaient réservées habituellement. Un autre aspect intéressant à remarquer est que la première génération de sages-femmes et infirmières transjordaniennes et palestiniennes incluait un nombre important de femmes chrétiennes, parce que les familles musulmanes étaient généralement moins enclines à laisser leurs filles étudier dans le domaine de la santé - ceci, malgré la fatwa émise en 1925 par le Haut Conseil islamique de Palestine autorisant les femmes musulmanes à aller dans les hôpitaux gouvernementaux. En dépit de la condamnation morale et sociale de leur profession durant la période mandataire, les sages-femmes et les infirmières transjordaniennes et palestiniennes réussirent à accéder à une nouvelle sphère sociale et à gagner un statut professionnel reconnu par les autorités. Certaines reçurent des bourses pour aller effectuer leurs études à l'étranger, participèrent à des conférences internationales et accédèrent à des postes importants dans les institutions. En d'autres termes, elles eurent accès à des espaces institutionnels qui étaient autrefois réservés aux hommes; purent entreprendre des voyages et séjourner à l'étranger seules et accéder à de nouvelles formes d'autonomie personnelle, sans être socialement ostracisées, malgré la résistance exprimée par la société locale. Ainsi, lorsque l'Emir Abdallah «ordonna au ministre de l'Education d'effectuer des inspections dans toutes les écoles féminines pour s'assurer que les enseignantes étaient en mesure d'assumer leurs 'responsabilités religieuses et éthiques'», ${ }^{47}$ il ne sembla pas se soucier des sages-femmes et des infirmières. A côté de la santé, l'éducation était le seul domaine dans lequel les femmes avaient la possibilité d'entreprendre une activité en dehors de l'espace domestique et d'entrer dans le marché du travail rémunéré, alors que jusqu'à l'époque coloniale les femmes en étaient généralement exclues. Il est possible que l'exclusion des soignantes des inspections soit due au fait que, à cette époque, il y avait très peu de femmes locales travaillant dans les hôpitaux et les dispensaires de l'Emirat, car le personnel était très majoritairement masculin. Comme mentionné au début, les quelques femmes présentes qui travaillaient dans les institutions missionnaires étaient d'habitude européennes - missionnaires protestantes britanniques, sœurs catholiques italiennes, femmes d'administrateurs coloniaux, etc. Il est aussi possible que, puisque les sages-femmes et les infirmières étaient perçues comme des spécialistes dans le domaine de la santé féminine et infantile, leurs activités aient été appréhendées comme moins subversives vis-à-vis de l'ordre social. En revanche, l'éducation des filles dans les écoles, des lieux institutionnels situés

47 Massad 2001, 89.

Gesnerus 75 (2018) 
à l'extérieur de la sphère familiale, introduisait de nouvelles pratiques dans le contexte transjordanien qui semblaient en mesure de menacer plus directement les structures familiales et les valeurs locales.

\section{Conclusion}

Le bref aperçu historique donné dans la première partie de cet article permet de saisir l'impact important joué par la colonisation britannique et l'entreprise missionnaire dans la construction des premières infrastructures sanitaires et dans la médicalisation de la procréation en Transjordanie. Préoccupées par la haute mortalité infantile, les autorités britanniques en collaboration avec les sociétés missionnaires ont posé les bases pour la pénétration de la biomédcine et la médicalisatioin de la santé maternelle et infantile dans l'Emirat. Dans le but de moderniser le système natal local, l'administration britannique d'une part a combattu les dayat menant des campagnes de délégitimation, limitant leur pratique et leur imposant le contrôle du personnel médical; d'autre part elle a créé les conditions permettant l'émergence d'un groupe de sages-femmes et infirmières transjordaniennes et palestiniennes pouvant occuper les postes créés dans les hôpitaux et centres de PMI jusqu'alors occupés exclusivement par du personnel européen. La médicalisation de la procréation s'est réalisée au cours de plusieurs décennies donnant lieu à des négociations asymmtétriques entre colonisateurs et colonisés, où les dayat et les mères, représententantes de ces derniers, ont dû progressivement se plier aux modèles et aux pratiques du système biomédical. À la fin de ce parcours, qui s'est terminé bien après l'indépendance, les accoucheuses ont disparu, laissant la place à un pluralisme médical dominé néanmoins par les institutions biomédicales. Les biographies de deux parmi les premières sages-femmes qui ont pratiqué en Transjordanie permettent d'analyser les effets de la colonisation sur les femmes trasjordaniennes et palestiniennes qui ont pu accéder à l'instruction et à des professions nouvelles. Malgré son origine coloniale et ses effets destructeurs, la médicalisation de la procréation a ainsi généré de nouvelles possibilités existentielles modifiant les relations familiales, les normes sociales et ouvrant aux femmes, même de condition modeste, de nouveaux horizons. 


\section{Bibliographie}

Abul Lughod, Lila (éd.), Remaking Women. Feminism and Modernity in the Middle East (Princeton 1998)

Al-Tutunji, Jamil, «Tarikh da'irat al-sahha fi al-Urdun», dans: Taqrir Sanawi li Wizarat al-Sahha 1951-57, Ministry of Health (Amman 1957)

Amadouni, Vartan, «Infrastructural development under British Mandate», in: Eugene Rogan et Tariq Tell (eds.), Village, Steppe and the State: The Social Origins of Modern Jordan (Londres 1994)

Amadouni, Vartan, «The campaign against malaria in Transjordan, 1926-1946: epidemiology, geography, and politics», Journal of the History of Medicine and Allied Sciences 52 (1997), 453-484

Annual Report of the Department of Health of Transjordan (Amman 1929; 1939)

Arnold, David, Colonizing the Body: State Medicine and Epidemic Disease in Nineteenth-century India (Berkeley/Los Angeles 1993)

Boddy, Janice, Civilizing Women. British Crusades in Colonial Sudan (Princeton 2007)

Bourmaud, Philippe, «Ya Doctor»: devenir médecin et exercer son art en «Terre sainte»: une expérience de pluralisme médical dans l'Empire ottoman finissant (1871-1918), Thèse de doctorat, Université Aix-Marseille I-Université de Provence (Aix-en-Provence 2007)

Granqvist, Hilma, Birth and Childhood among the Arabs: Studies in a Muhammadan Village in Palestine (Helsingfors 1947)

Hugon, Anne, «Les sages-femmes africaines en contexte colonial: auxiliaires de l'accouchement ou agents de la médicalisation? Le cas du Ghana, des années 1930 aux années 1950», dans: Patrice Bourdelais/Olivier Faure (éds.), La diffusion de nouvelles pratiques de santé: acteurs, dynamiques, enjeux (17ème-19 ${ }^{\text {ème }} \mathrm{s}$.) (Paris 2005) 175-193

Hugon, Anne, «Réformer la maternité: un projet colonial. Gold Coast, des années 1920 aux années 1950» (2011) Publication en ligne: http://www.societe-histoirenaissance.fr/spip.php?article66

Inhorn, Marcia, Infertility and Patriarchy: The Cultural Politics of Gender and Family Life in Egypt (Philadelphie 1996)

Jaussen, Antonin, Coutumes des Arabes au pays de Moab (Paris 1908)

Jaussen, Antonin, Naplouse et son district (Paris 1927)

Jaussen, Antonin, Coutumes palestinienne: Naplouse et son district (Paris 1927)

Jolly, Margaret/Kalpana (éds.) Maternities and Modernities: Colonial and Postcolonial Experiences in Asia and the Pacific (Cambridge/New York 1998)

Jordan, Birgitte, Birth in Four Cultures: A Cross-Cultural Investigation of Childbirth in Yucatan, Holland, Sweden and the United States (Long Grove 1993)

Koven, Seth/Sonya Michel, Mothers of a New World: Maternalist Politics and the Origins of Welfare States (New York 1993)

Maffi, Irene, Women, Health and the State in the Middle East. Politics and practices of childbirth in Jordan (Londres 2012)

Massad, Joseph, Colonial Effects: The Making of National Identity in Jordan (New York 2001)

Munif, Abdul-Rahman, Story of a City: A Childhood in Amman (Londres 1998)

Gesnerus 75 (2018) 
Quattrocchi, Patrizia, Corpo, riproduzione e salute tra le donne maya dello Yucatan (Messico) (Pise 2011)

Rapport Administratif Annuel pour la Palestine et la Transjordanie (Jérusalem 1921, 1922, 1925, 1931, 1932, 1934, 1937, 1938, 1939)

Rogan, Eugene/Tariq Tell (éds.), Village, Steppe and the State: The Social Origins of Modern Jordan (Londres 1994)

Rogan, Eugene, Frontiers of the State in Late Ottoman Empire. Transjordan, 18501921 (Cambridge 1999)

Sargent, Carolyn/Grace Bascope, «Ways of knowing about birth in three cultures», Medical Anthropology Quarterly 10 (1996) 213-236

Shepherd, Naomi, Ploughing Sand: British Rule in Palestine, 1917-1948 (Londres 1999)

Sultan, Fathi A., Al-tamrid fi al-Urdun: al-nush, al-tatawur, al-tumuh, Minsitry of Culture (Amman 1998)

Summers, Carol, «Intimate colonialism: the imperial production of reproduction in Uganda», Women, Family, State, and Economy in Africa 16 (1991) 787-897

Tbaishat, Muhammad, The Relationship between Modern and Traditional Medical Systems: A Case-study from Northern Jordan, Thèse de doctorat, Yarmouk University (Irbid 1991)

Van Hollen, Cecilia, Birth on the Treshold. Childbirth and Modernity in South India (Berkeley 2003)

Van Tol, Deanne, «Mothers, babies, and the colonial state: the introduction of maternal and infant welfare services in Nigeria», Spontaneous Generations 1 (2007) 110-31

Wilson, Mary, King Abdallah, Britain and the Making of Jordan (Cambridge 1987)

Young, Elise, Between Daya and Doctor: A History of the Impact of Modern Nation-state Building on Health East and West of the Jordan River, Thèse de doctorat, University of Massachusetts, (Amherst 1997) 